UDC 512.5

B. Nisanci, A. Pancar (Ondokuz Mayıs Univ., Turkey)

\title{
ON GENERALIZATION OF $\oplus$-COFINITELY SUPPLEMENTED MODULES
}

\section{ПРО УЗАГАЛЬНЕННЯ $\bigoplus$-КОФІНІТНО ПОПОВНЕНИХ МОДУЛІВ}

We study the properties of $\oplus$-cofinitely radical supplemented modules or briefly $c g s^{\oplus}$-modules. It is shown that: a module with Summand Sum Property (SSP) is $c g s^{\oplus}$ if and only if $M / w \operatorname{Loc}^{\oplus} M\left(w \operatorname{Loc}^{\oplus} M\right.$ is the sum of all $w$-local direct summands of a module $M$ ) does not contain any maximal submodule; every cofinite direct summand of a UC-extending $c g s^{\oplus}$-module is $c g s^{\oplus}$; for any ring $R$, every free $R$-module is $c g s^{\oplus}$ if and only if $R$ is semiperfect.

Досліджено властивості $\oplus$-кофінітно радикальних поповнених модулів або скорочено $\mathrm{cgs}^{\oplus}$-модулів Показано, що модуль із властивістю суми доданків SSP є cgs ${ }^{\oplus}$-модулем тоді і тільки тоді, коли $M / w \operatorname{Loc}^{\oplus} M\left(w \operatorname{Loc}^{\oplus} M-\right.$ сума всіх $w$-локальних прямих доданків модуля $\left.M\right)$ не містить жодного максимального субмодуля; кожний прямий доданок UC-розширюваного $\mathrm{cgs}^{\oplus}$-модуля $\epsilon \mathrm{cgs}^{\oplus}$-модулем; для будь-якого кільця $R$ кожний вільний $R$-модуль є $c g s^{\oplus}$-модулем тоді і тільки тоді, коли $R \epsilon$ напівперфектним.

1. Introduction. In this note $R$ will be an associative ring with identity and all modules are unital left $R$-modules. Let $M$ be an $R$-module. The notation $N \subseteq M$ means that $N$ is a submodule of $M . \operatorname{Rad} M$ will indicate Jacobson radical of $M$. A submodule $N$ of an $R$-module $M$ is called small in $M$ (notation $N \ll M$ ), if $N+L \neq M$ for every proper submodule $L$ of $M$. Let $M$ be an $R$-module and let $N$ and $K$ be any submodules of $M . K$ is called a supplement of $N$ in $M$ if $M=N+K$ and $N \cap K \ll K$ (see [1]). Following [1], $M$ is called supplemented if every submodule of $M$ has a supplement in $M$. A submodule $N$ of a module $M$ is called cofinite in $M$ if the factor module $\frac{M}{N}$ is finitely generated. A module $M$ is called cofinitely supplemented if every cofinite submodule of $M$ has a supplement in $M$ (see [2]). Clearly supplemented modules are cofinitely supplemented. A module $M$ is called $\oplus$-supplemented if every submodule of $M$ has a supplement that is a direct summand of $M$ (see [3]). As a proper generalization of $\oplus$-supplemented modules, the notation of $\oplus$-cofinitely supplemented modules was introduced by Calisici and Pancar [4]. A module $M$ is called $\oplus$-cofinitely supplemented if every cofinite submodule of $M$ has a supplement that is a direct summand of $M$. Also, finitely generated $\oplus$-cofinitely supplemented modules are $\oplus$-supplemented.

In [5] (Theorem 10.14), another generalization of supplement submodule was called as radical supplement or briefly Rad-supplement (according to [6], generalized supplement). For a module $M$ and a submodule $N$ of $M$, a submodule $K$ of $M$ is called a Rad-supplement of $N$ in $M$ if $N+K=M$ and $N \cap K \subseteq \operatorname{Rad} K$. An $R$-module $M$ is called radical supplemented or briefly Rad-supplemented if every submodule of $M$ has a Rad-supplement in $M$ (in [6], generalized supplemented or GS-module). Since the Jacobson radical of a module is sum of all small submodules, every supplement is a Rad-supplement. Therefore every supplemented module is Rad-supplemented. In [7], $M$ is called cofinitely radical supplemented or briefly cofinitely Rad-supplemented if every cofinite submodule of $M$ has a Rad-supplement in $M$. Clearly Rad-supplemented modules are cofinitely Rad-supplemented. 
Let $M$ be an $R$-module. $M$ is called $\oplus$-radical supplemented or briefly $\oplus$-Radsupplemented or generalized $\oplus$-supplemented if every submodule of $M$ has a Radsupplement that is a direct summand of $M$. Clearly $\oplus$-Rad-supplemented modules are Rad-supplemented. A module $M$ is called $\oplus$-cofinitely radical supplemented (according to [8], generalized $\oplus$-cofinitely supplemented) if every cofinite submodule of $M$ has a Rad-supplement that is a direct summand of $M$. Instead of a $\oplus$-cofinitely radical supplemented module, we will use a $c g s^{\oplus}$-module.

In this paper we study the properties of $\mathrm{cgs}^{\oplus}$-modules as both a proper generalization of $\oplus$-Rad-supplemented modules and a generalization of $\oplus$-cofinitely supplemented modules. We prove that a module $M$ with SSP is $c g s^{\oplus}$ if and only if $M / w \operatorname{Loc}^{\oplus} M$ does not contain any maximal submodule, where $w \operatorname{Loc}^{\oplus} M$ is the sum of all $w$-local direct summands of $M$. Also we show that any direct sum of $c g s^{\oplus}$-modules is also a $c g s^{\oplus}$-module. Using the mentioned fact we give a characterization of semiperfect rings.

2. Some properties of $\oplus$-cofinitely radical supplemented modules. It is clear that every $\oplus$-cofinitely supplemented module is $c g s^{\oplus}$, but it is not generally true that every $\mathrm{cgs}^{\oplus}$-module is $\oplus$-cofinitely supplemented. Later we shall give an example of such modules (see Example 2.1). Now we give an analogue of these modules.

Proposition 2.1. Let $M$ be a cgs $^{\oplus}$-module with small radical. Then $M$ is $\oplus$ cofinitely supplemented.

Proof. Let $U$ be any cofinite submodule of $M$. By the hypothesis, there exist submodules $V, V^{\prime}$ of $M$ such that $M=U+V, U \cap V \subseteq \operatorname{Rad} V$ and $M=V \oplus V^{\prime}$. Since $U \cap V \subseteq \operatorname{Rad} V \subseteq \operatorname{Rad} M \ll M$ and $V$ is a direct summand of $M$, then $U \cap V \ll V$ by [1] (19.3.(5)). Hence $M$ is $\oplus$-cofinitely supplemented.

Let $M$ be an $R$-module. If every proper submodule of $M$ is contained a maximal submodule of $M, M$ is called coatomic. Note that every coatomic module has small radical.

Corollary 2.1. Let $M$ be a coatomicR-module. Then $M$ is a $\mathrm{cgs}^{\oplus}$-module if and only if it is $\oplus$-cofinitely supplemented.

Every $c g s^{\oplus}$-module is cofinitely Rad-supplemented but the converse is not true. For example, a left (cofinitely) Rad-supplemented ring which is not supplemented (i.e., semiperfect) is cofinitely Rad-supplemented over itself, but not a $\mathrm{cgs} s^{\oplus}$-module.

Therefore we have the following implications on modules:

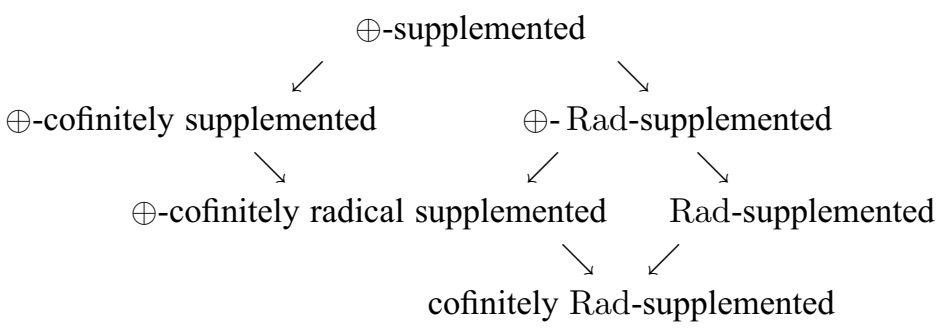

We begin by some general properties of $c g s^{\oplus}$-modules. To prove that any direct sum of $c g s^{\oplus}$-modules is $c g s^{\oplus}$, we use the following standart Lemma ([7], 3.4).

Lemma 2.1. Let $M$ be an R-module and $N, U$ be submodules of $M$ such that $N$ is cofinitely Rad-supplemented, $U$ cofinite and $N+U$ has a Rad -supplement $A$ in $M$. Then $N \cap(U+A)$ has a Rad -supplement $B$ in $N$ and $A+B$ is a Rad-supplement of $U$ in $M$. 
Proof. Let $A$ be a Rad-supplement of $N+U$ in $M$. Then

$$
\frac{N}{N \cap(U+A)} \cong \frac{N+U+A}{U+A} \cong \frac{M / U}{(U+A) / U}
$$

Since $U$ is a cofinite submodule of $N, N \cap(U+A)$ is cofinite. By hypothesis, $N$ is cofinitely Rad-supplemented, $N \cap(U+A)$ has a Rad-supplement $B$ in $N$. Then $M=(N+U)+A=U+A+B$ and by [1] (19.3), $U \cap(A+B) \subseteq A \cap(U+B)+$ $+B \cap(U+A) \subseteq A \cap(N+U)+B \cap(U+A) \subseteq \operatorname{Rad}(A+B)$. Therefore $A+B$ is a Rad-supplement of $U$ in $M$.

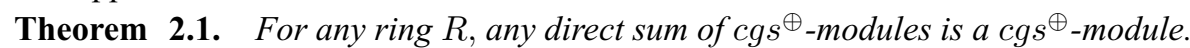

Proof. Let $R$ be any ring and $\left\{M_{i}\right\}_{i \in I}$ be any family of $c g s^{\oplus}$-modules. Let $M=$ $=\oplus_{i \in I} M_{i}$ and $N$ be a cofinite submodule of $M$. Then $M=\oplus_{j=1}^{n} M_{i_{j}}+N$ and it is clear that $\{0\}$ is $\operatorname{Rad}$-supplement of $M=M_{i_{1}}+\left(\oplus_{j=2}^{n} M_{i_{j}}+N\right)$. Since $M_{i_{1}}$ is a cgs ${ }^{\oplus}$-module, $M_{i_{1}} \cap\left(\oplus_{j=2}^{n} M_{i_{j}}+N\right)$ has a Rad-supplement $V_{i_{1}}$ in $M_{i_{1}}$ such that $V_{i_{1}}$ is a direct summand of $M_{i_{1}}$. By Lemma 2.1, $V_{i_{1}}$ is a Rad-supplement of $\oplus_{j=2}^{n} M_{i_{j}}+N$ in $M$. Note that since $M_{i_{1}}$ is a direct summand of $M, V_{i_{1}}$ is also a direct summand of $M$. By repeated use of Lemma 2.1, since the set $J$ is finite at the end we will obtain that $N$ has a Rad-supplement $V_{i_{1}}+V_{i_{2}}+\ldots+V_{i_{r}}$ in $M$ such that every $V_{i_{j}}, 1 \leq j \leq n$, is a direct summand of $M_{i_{j}}$. Since every $M_{i_{j}}$ is a direct summand of $M, \sum_{j=1}^{n} V_{i_{j}}=\oplus_{j=1}^{n} V_{i_{j}}$ is a direct summand of $M$. Hence $M$ is a $\operatorname{cgs}^{\oplus}$-module.

Recall from [7] that a module $M$ is called $w$-local if it has a unique maximal submodule. It is clear that a module is $w$-local if and only if its radical is maximal.

Local modules are $w$-local. But it is not generally true that every $w$-local module is local. For example, $\mathrm{p}$ any prime, the $\mathbb{Z}$-module $\mathbb{Q} \oplus \mathbb{Z}_{\mathrm{p}}$ is $w$-local but it is not local. It is trivial that $w$-local modules are a generalization of local modules. This fact plays a key role in our working.

Proposition 2.2. The following statements are equivalent for a $w$-local module $M$.

(i) $\operatorname{Rad} M \ll M$.

(ii) $M$ is finitely generated.

Proof. Suppose that $M$ is a $w$-local module. Then $\operatorname{Rad} M$ is a maximal submodule of $M$. Thus $\operatorname{Rad} M+R m=M$ for every $m \in M \backslash \operatorname{Rad} M$. Since $\operatorname{Rad} M \ll M$, then $R m=M$. Hence $M$ is finitely generated. The converse is clear.

Proposition 2.3. Let $M$ be a w-local R-module. Then $M$ is a cgs $^{\oplus}$-module.

Proof. It follows from [7] (Lemma 3.2).

Proposition 2.4. Let $M$ be a cgs $^{\oplus}{ }^{\oplus}$-module. If $M$ has a maximal submodule, then $M$ contains a w-local direct summand.

Proof. Let $L$ be a maximal submodule of $M$. Then $L$ is cofinite and it follows that there exist $K, K^{\prime}$ submodules of $M$ such that $L+K=M, L \cap K \subseteq \operatorname{Rad} K$ and $M=K \oplus K^{\prime}$. By Lemma 3.3 in [7], $K$ is $w$-local. Hence $K$ is a $w$-local direct summand of $M$.

Let $M$ be an $R$-module. $w \operatorname{Loc}^{\oplus} M$ will denote the sum of all $w$-local direct summands of $M$.

Recall from [1] that an $R$-module $M$ has Summand Sum Property (SSP) if the sum of two direct summands of $M$ is again a direct summand of $M$.

We give a characterization of $c g s^{\oplus}$-modules. Firstly we need the following lemma which is a generalization of [2] (Lemma 2.9). 
Lemma 2.2. Let $M$ be an R-module and $N$ be a cofinite submodule of $M$. Let $\left\{L_{i}\right\}_{i=1}^{n}$ be the family of $w$-local submodules such that $K$ is a Rad-supplement of $N+L_{1}+\ldots+L_{n}$ in $M$. Then $K+\sum_{i \in I} L_{i}$ is a Rad-supplement of $N$ in $M$ such that $I$ is a subset of $\{1,2, \ldots, n\}$.

Proof. Suppose that $n=1$. Consider the submodule $H=(N+K) \cap L_{1}$ of $L_{1}$. $K$ is a Rad-supplement of $N+L_{1}$, so that $M=N+L_{1}+K$ and $\left(N+L_{1}\right) \cap K \subseteq \operatorname{Rad} K$. Then $H$ is a cofinite submodule of $L_{1}$. Since $L_{1}$ is $w$-local, then $\operatorname{Rad} L_{1}$ is a unique maximal submodule of $L_{1}$. Note that $H \subseteq \operatorname{Rad} L_{1}$. By [9] (19.3), $N \cap\left(K+L_{1}\right) \subseteq$ $\subseteq K \cap\left(N+L_{1}\right)+H \subseteq \operatorname{Rad} K+\operatorname{Rad} L_{1} \subseteq \operatorname{Rad}\left(K+L_{1}\right)$. Therefore $K+L_{1}$ is a Rad-supplement of $N$. This proves the result when $n=1$. Suppose that $n \geq 2$. By induction on $n$, there exists a subset $I^{\prime}$ of $\{2,3, \ldots, n\}$ such that $K+\sum_{i \in I^{\prime}} L_{i}$ is a Rad-supplement of $N+L_{1}$ in $M$. Now the case $n=1$ shows that $K+L_{1}+\sum_{i \in I^{\prime}} L_{i}$ is a Rad-supplement of $N$ in $M$.

Theorem 2.2. Let $R$ be any ring and $M$ be an $R$-module with $S S P$. Then the following statements are equivalent.

(i) $M$ is a $\mathrm{cgs}^{\oplus}$-module.

(ii) Every maximal submodule of $M$ has a Rad-supplement that is a direct summand of $M$.

(iii) $M / w \operatorname{Loc}^{\oplus} M$ does not contain a maximal submodule.

Proof. (i) $\Rightarrow$ (ii) Clear.

(ii) $\Rightarrow$ (iii). Suppose that $M / w \operatorname{Loc}^{\oplus} M$ contains a maximal submodule $U / w \operatorname{Loc}^{\oplus} M$. Then $U$ is a maximal submodule of $M$. By assumption, $U$ has a $\operatorname{Rad}$-supplement $V$ that is a direct summand of $M$. Then $V$ is $w$-local and it follows that $V \subseteq w \operatorname{Loc}^{\oplus} M$. Since $M=U+V$ and $w \operatorname{Loc}^{\oplus} M \subseteq U$, we get $M=U$ which is a contradiction.

(iii) $\Rightarrow$ (i). Let $N$ be any cofinite submodule of $M$. Then $N+w \operatorname{Loc}^{\oplus} M$ is a cofinite submodule of $M$. By (iii), $M=N+w \operatorname{Loc}^{\oplus} M$. Because $M / N$ is finitely generated, there exist $w$-local submodules $L_{i}, 1 \leq i \leq n$, for some positive integer $n$, such that each of them is a direct summand of $M$ and $M=N+\sum_{i=1}^{n} L_{i}$ has a Rad-supplement $\{0\}$ in $M$. By Lemma 2.2, $\sum_{i \in I \prime} L_{i}$ is a Rad-supplement of $N$ in $M$ such that $I^{\prime}$ is a subset of $\{1,2, \ldots, n\}$. Moreover $\sum_{i \in I} L_{i}$ is a direct summand of $M$. Thus $M$ is a $c g s^{\oplus}$-module.

Example 2.1. Let $R$ be a commutative local ring which is not a valuation ring. Let $x$ and $y$ be elements of $R$, neither of them divides the other. By taking a suitable quotient ring, we may assume that $(x) \cap(y)=0$ and $x I=y I=0$, where $I$ is the maximal ideal of $R$. Let $F$ be a free module with generators $a_{1}, a_{2}, a_{3}$. Let $N$ be the submodule generated by $x a_{1}-y a_{2}$ and $M=F / N$. $R$ is local, so ${ }_{R} R$ is a $\mathrm{cgs}^{\oplus}$-module. By Theorem 2.1, F is a $c g s^{\oplus}$-module. Suppose that $M$ is a $\mathrm{cgs}^{\oplus}$-module. Since $F$ is finitely generated, $M$ is finitely generated and it follows that $M$ has a small radical. By Proposition 2.1, $M$ is $\oplus$-(cofinitely) supplemented. This is a contradiction by [10] (Example 2.3).

This example shows that the factor module of a $\mathrm{cgs}^{\oplus}$-module is not in general $\mathrm{cgs}^{\oplus}$.

Let $R$ be a ring and $M$ be an $R$-module. We consider the following condition.

$\left(D_{3}\right)$ If $K$ and $N$ are direct summands of $M$ with $M=K+N$, then $K \cap N$ is also a direct summand of $M$ (see [11]). 
Proposition 2.5. Let $M$ be a $\mathrm{cgs}^{\oplus}$-module with $\left(D_{3}\right)$. Then every cofinite direct summand of $M$ is a $\mathrm{cgs}^{\oplus}$-module.

Proof. Let $N$ be any cofinite direct summand of $M$. Then there exists a submodule $N^{\prime}$ of $M$ such that $M=N \oplus N^{\prime}$ and $N^{\prime}$ is finitely generated. Let $U$ be any cofinite submodule of $N$. Note that $M / U \cong N / U \oplus N^{\prime}$ is finitely generated so that $U$ is also cofinite submodule of $M$. Since $M$ is a $c g s^{\oplus}$-module, then there exists a direct summand $V$ of $M$ such that $M=U+V$ and $U \cap V \subseteq \operatorname{Rad} V$. Hence $N=U+(N \cap V)$. Since $M$ has $\left(D_{3}\right), N \cap V$ is a direct summand of $M$. Furthermore $N \cap V$ is a direct summand of $N$ because $N$ is a direct summand of $M$. Then $U \cap(N \cap V)=U \cap V \subseteq \operatorname{Rad} M$. Note that $U \cap(N \cap V) \subseteq \operatorname{Rad}(N \cap V)$ by [1] (19.3). Hence $N$ is a $c g s^{\oplus}$-module.

Corollary 2.2. Let $M$ be a $U C$-extending module. If $M$ is a cgs $^{\oplus}$-module, then every cofinite direct summand of $M$ is a $\mathrm{cgs}^{\oplus}{ }^{\oplus}$-module.

Recall from [1] that a submodule $U$ of an $R$-module $M$ is called fully invariant if $f(U)$ is contained in $U$ for every $R$-endomorphism $f$ of $M$. Let $M$ be an $R$-module and $\tau$ be a preradical for the category of $R$-modules. Then, $\operatorname{Rad} M$ and $\tau(M)$ are fully invariant submodule of $M$. An $R$-module $M$ is called a (weak) duo module if every (direct summand) submodule of $M$ is fully invariant. Note that weak duo modules has $S S P$ (see [9]).

Corollary 2.3. Let $R$ be a ring and $M$ be a weak duo $R$-module. Then $M$ is a cgs ${ }^{\oplus}$-module if and only if every maximal submodule of $M$ has a Rad-supplement that is a direct summand of $M$.

Proposition 2.6. Let $M$ be a $\mathrm{cgs}^{\oplus}{ }^{\oplus}$-module and $U$ be a fully invariant submodule of $M$. Then $M / U$ is a cgs ${ }^{\oplus}$-module.

Proof. Let $K / U$ be a cofinite submodule of $M / U$. Then $K$ is a cofinite submodule of $M$. Since $M$ is a $c g s^{\oplus}$-module, then $(N+U) / U$ is a Rad-supplement of $K / U$ in $M / U$ by [6] (Proposition 2.6) and $M=N \oplus N^{\prime}$ for $N^{\prime}$ is a submodule of $M$. By hypothesis, $U$ is a fully invariant submodule of $M$. Note that $U=(U \cap N) \oplus\left(U \cap N^{\prime}\right)$ by [9] (Lemma 2.1). Then $M / U=(N+U) / U \oplus\left(N^{\prime}+U\right) / U$. $(N+U) / U$ is a Rad-supplement of $K / U$ such that $(N+U) / U$ is a direct summand of $M / U$. Hence $M / U$ is a $c g s^{\oplus}$-module.

Corollary 2.4. Let $M$ be a cgs $^{\oplus}$-module. Then $M / \operatorname{Rad} M$ and $M / \tau(M)$ is a cgs ${ }^{\oplus}$-module.

Proposition 2.7. Let $M$ be a $\mathrm{cgs}^{\oplus}$-module and $U$ be a fully invariant submodule of $M$. If $U$ is a cofinite direct summand of $M$, then $U$ is a $\mathrm{cgs}^{\oplus}$-module.

Proof. Let $U$ be a cofinite submodule of $M$. Since $U$ is a cofinite direct summand of $M$, it follows that $U \oplus U^{\prime}=M$ for $U^{\prime} \subseteq M$. Let $V$ be a cofinite submodule of $U$. Then $U / V$ and $U^{\prime}$ is finitely generated. Therefore $V$ is a cofinite submodule of $M$. By hypothesis, $V+K=M, V \cap K \subseteq \operatorname{Rad} K$ and $M=K \oplus K^{\prime}$ such that $K, K^{\prime} \subseteq M$. Note that $U=(U \cap K) \oplus\left(U \cap K^{\prime}\right)$ by [9] (Lemma 2.1). Then $U=V \oplus(U \cap K)$ and $V \cap(U \cap K) \subseteq \operatorname{Rad} M$. Since $U \cap K$ is a direct summand of $M$, then $V \cap(U \cap K) \subseteq \operatorname{Rad}(U \cap K) . U \cap K$ is a $\operatorname{Rad}$-supplement of $V$ in $U$ that is a direct summand of $U$. It follows that $U$ is a $\mathrm{cgs}^{\oplus}$-module.

Let $\left\{L_{i}\right\}_{i \in I}$ be the family of $\mathrm{cgs}^{\oplus}$-submodules of $M . C g s^{\oplus} M$ will denote the sum of $L_{i} s$ for all $i \in I$. That is $C g s^{\oplus} M=\sum_{i \in I} L_{i}$. It is clear that $w \operatorname{Loc}^{\oplus} M \subseteq C g s^{\oplus} M$.

Proposition 2.8. Let $R$ be a ring, $M$ be an $R$-module and every cgs $^{\oplus}{ }^{\oplus}$-submodule of $M$ be a direct summand of $M$. Then every maximal submodule of $M$ has a Rad- 
supplement that is a direct summand of $M$ if and only if $M / C g s^{\oplus} M$ does not contain a maximal submodule.

Proof. $(\Rightarrow)$ Suppose that $M / C g s^{\oplus} M$ contains a maximal submodule $U / C g s^{\oplus} M$. Then $U$ is a maximal submodule of $M$. By assumption, there exist $V, V^{\prime}$ submodules of $M$ such that $U+V=M, U \cap V \subseteq \operatorname{Rad} V$ and $M=V \oplus V^{\prime}$. By [7] (Lemma 3.3) $V$ is $w$-local. Then $V$ is a $c g s^{\oplus}$-module by Proposition 2.3. It follows that $V \subseteq C g s^{\oplus} M$. $M / C g s^{\oplus} M=U / C g s^{\oplus} M$, so that $M=U$ which is a contradiction.

$(\Leftarrow)$ Let $P$ be a maximal submodule of $M$. By assumption, $P$ does not contain $C g s^{\oplus} M$. Hence there exists a $\mathrm{cgs}^{\oplus}$-module $L$ of $M$ such that it is not a submodule of $P$ is a maximal submodule of $M$ and $L \nsubseteq P$, then $M=P+L$. Note that $M / P \cong$ $\cong L /(P \cap L)$. It follows that $P \cap L$ is a maximal submodule of $L$. Then $P \cap L$ is a cofinite submodule of $L$. By assumption, there exist $X, X^{\prime}$ submodules of $M$ such that $L=(P \cap L)+X,(P \cap L) \cap X \subseteq \operatorname{Rad} X$ and $L=X \oplus X^{\prime}$. It follows that $M=P+X$ and $P \cap X \subseteq \operatorname{Rad} X$. Moreover by hypothesis, $X$ is a direct summand of $M$. Therefore $P$ has a Rad-supplement that is a direct summand of $M$.

Theorem 2.3. Let $M$ be an R-module such that $M=M_{1} \oplus M_{2}$ is a direct sum of submodules $M_{1}, M_{2}$. Then $M_{2}$ is a $\mathrm{cgs}^{\oplus}{ }^{\oplus}$ module if and only if there exists a submodule $K$ of $M_{2}$ such that $K$ is a direct summand of $M, M=K+N$ and $N \cap K \subseteq \operatorname{Rad} K$ for every cofinite submodule $N / M_{1}$ of $M / M_{1}$.

Proof. $(\Rightarrow)$ Let $N / M_{1}$ be any cofinite submodule of $M / M_{1}$. Then $N$ is a cofinite submodule of $M$ and it follows that $N \cap M_{2}$ is a cofinite submodule of $M_{2}$. By hypothesis, there exist $K, K^{\prime}$ submodules of $M_{2}$ such that $M_{2}=\left(N \cap M_{2}\right)+K$, $\left(N \cap M_{2}\right) \cap K \subseteq \operatorname{Rad} K$ and $M_{2}=K \oplus K^{\prime}$. Note that $M=N+K$ and $N \cap K \subseteq \operatorname{Rad} K$. Since $K$ is a direct summand of $M_{2}$, then $K$ is a direct summand of $M$.

$(\Leftarrow)$ Let $U$ be any cofinite submodule of $M_{2}$. Then $M_{2} / U$ is finitely generated. It follows that $\left(U+M_{1}\right) / M_{1}$ is a cofinite submodule of $M / M_{1}$. By hypothesis, there exists a submodule $K$ of $M_{2}$ such that $K$ is a direct summand of $M, M=K+U+M_{1}$ and $\left(U+M_{1}\right) \cap K \subseteq \operatorname{Rad} K$. It follows that $M_{2}=U+K$ and $U \cap K \subseteq \operatorname{Rad} K$. Therefore $M_{2}$ is a $\mathrm{cgs}^{\oplus}$-module.

A ring $R$ is semiperfect if $R / \operatorname{Rad} R$ is semisimple and idempotents can be lifted modulo $\operatorname{Rad} R$. It is shown [4] (Theorem 2.9) that $R$ is semiperfect if and only if ${ }_{R} R$ is $\oplus$-supplemented if and only if every free $R$-module is $\oplus$-cofinitely supplemented. Now we generalize this fact.

Theorem 2.4. Let $R$ be any ring. Then $R$ is semiperfect if and only if every free $R$-module is a $\mathrm{cgs}^{\oplus}$-module.

Proof. Let $F$ be any free $R$-module. Since $R$ is semiperfect, then ${ }_{R} R$ is $\oplus$-cofinitely supplemented and it follows that ${ }_{R} R$ is a $c g s^{\oplus}$-module. By Theorem $2.1, F$ is a $c g s^{\oplus}{ }_{-}$ module. Conversely, suppose that every free $R$-module is $\mathrm{cgs}^{\oplus}$. Then ${ }_{R} R$ is a $\mathrm{cgs} \mathrm{s}_{-}$ module. By Proposition 2.1, ${ }_{R} R$ is (cofinitely) $\oplus$-supplemented, i.e., $R$ is semiperfect.

Finally, we give an example of module, which is $\mathrm{cgs}^{\oplus}$ but not $\oplus$-cofinitely supplemented.

Example 2.2 (see [12], Theorem 4.3 and Remark 4.4). Let $M$ be a biuniform module and $S=$ End $(M)$. Suppose that $P$ is the projective $S$-module with $\operatorname{dim}(P)=$ $=(1,0)$. Then $P$ is a indecomposable $w$-local module. Since $\operatorname{dim}(P)=(1,0), P$ is not finitely generated. Hence $P$ is a $c g s^{\oplus}$-module but not $\oplus$-cofinitely supplemented. 
1. Wisbauer R. Foundations of module and ring theory. - Philadelphia: Gordon and Breach, 1991.

2. Alizade R., Bilhan G., Smith P. F. Modules whose maximal submodules have supplements // Communs Algebra. - 2001. - 29, № 6. - P. 2389-2405.

3. Harmancr A., Keskin D., Smith P. F. On $\oplus$-supplemented modules // Acta math. hungar. - 1999. - 83, № 1-2. - P. $161-169$.

4. Çalışıcı H., Pancar A. $\oplus$-Cofinitely supplemented modules // Chech. Math. J. - 2004. - 54, № 129. P. $1083-1088$.

5. Clark J., Lomp C., Vajana N., Wisbauer R. Lifting modules. - Basel etc.: Birkhäuser Verlag, 2006.

6. Wang Y., Ding N. Generalized supplemented modules // Taiwan. J. Math. - 2006. - 10, № 6. - P. $1589-$ 1601.

7. Büyükaşık E., Lomp C. On a recent generalization of semiperfect rings // Bull. Austral. Math. Soc. 2008. - 78. - P. $317-325$.

8. Koşan M. T. Generalized cofinitely semiperfect modules // Int. Electron. J. Algebra. - 2009. - 5. P. $58-69$.

9. Özcan A. C.., Harmancı A., Smith P. F. Duo modules // Glasgow Math. J. Trust. - 2006. - 48. P. 533-545.

10. Idelhadj A., Tribak R. On some properties of $\oplus$-supplemented modules // Int. J. Math. and Math. Sci. 2003. - 69. - P. 4373 - 4387.

11. Mohamed S. H., Müller B. J. Continuous and discrete modules // London Math. Soc. - Cambridge: Cambridge Univ. Press, 1990. - 147.

12. Puninski G. Projective modules over the endomorphism ring of a biuniform module // J. Pure and Appl. Algebra. - 2004. - 188. - P. 227 - 246.

Received 05.05.09 